\title{
EDITORIAL
}

\section{Educación médica actual: un reto conceptual}

\author{
Adalberto Amaya A.
}

\begin{abstract}
Existen múltiples factores que nos deben llevar a hacer una reflexión muy seria acerca de la educación médica actual y nuestro papel dentro de la misma. Si bien la tendencia universal está dada hacia la estandarización de los procesos de formación en búsqueda de una unidad que favorezca la homologación profesional con tendencia a la universalidad de los mismos, existe algo que no podemos desconocer en su construcción como son las necesidades sociales de contexto, la identidad y la tradición cultural. Es por esto que no solamente debemos tener en cuenta el cambio que se ha dado en la educación médica a nivel mundial sino esforzarnos haciendo un diagnóstico reflexivo de nuestra participación en el cambio y construir nuevas aspiraciones de formación en los estudiantes (curriculum), basados en una reflexión sistémica en nuestro contexto actual.
\end{abstract}

Si bien tenemos una tradición que marca una trayectoria y desempeño en cualquiera de los campos del quehacer humano, no podemos olvidar la importancia del cambio en el progreso de las culturas.

La educación médica no puede estar ajena a la evaluación de las necesidades de cambio, con pertinencia de acuerdo a los requerimientos sociales, universales, disciplinares y a las respuestas que exigen las necesidades actuales del ejercicio profesional en diferentes contextos socioculturales.

Al pensar en este cambio educativo, debemos tener presente que estamos formando estudiantes que salen a trabajar en un sistema de salud con una problemática individual, familiar y social cambiante, con un significativo y por que no decirlo, drástico cambio en la forma de ejercicio profesional, quien debe trabajar cada vez con una mayor presión laboral (de rendimiento y productividad), emocional (de calidad), económica (teniendo que cumplir en varios sitios de trabajo para suplir sus necesidades básicas), legal (por el riesgo a las demandas que se incrementa cada día más debido a que puede ser censurado por los pacientes quienes acceden fácilmente a la tecnología informática y tienen herramientas para discutir con el médico el tratamiento debido a la información que obtuvieron por ejemplo en la medicina basada en la evidencia) y con una exigencia conceptual que escapa a cualquier dimensión humana debido al avance horario de la ciencia y el conocimiento inmediato de dichos avances a través del acceso tecnológico lo cual supera cualquier capacidad individual. 
A lo anterior podemos agregar la dificultad que tenemos actualmente en la educación médica con respecto al cambio en los estudiantes que recibimos, los cuales manejan herramientas tecnológicas que los mantienen en contacto directo con todo el mundo en un solo "clic" del computador y les permite al igual que el paciente en muchas oportunidades censurar al docente por su falta de conocimiento actualizado, puesto que en muchas oportunidades, poseen una base informática activa por wifi, donde les brinda un manejo terapéutico ideal el cual puede muchas veces apoyarlos para censurar el tratamiento que da el docente al lado del paciente por no tener por ejemplo una evidencia tipo " $\mathrm{A}$ " en la medicina basada en la evidencia (MBE); en cuanto a lo anterior, es muy importante que tengamos claridad en cuanto a que la MBE, es una herramienta más de trabajo que deben conocer los estudiantes pero a la cual no podemos limitar la totalidad del manejo médico-quirúrgico del paciente, simplemente nos ayuda a definir ciertos criterios de dualidad pero no es en ella en quien debo encontrar la respuesta a la problemática de los pacientes; es con base en este criterio que debemos entender la importancia de preservar valores académicos como lo tiene la semiología y el diagnóstico clínico en la formación médica actual, herramientas que a corto tiempo se cree, sean difíciles de remplazar en un computador debido a la falta de sensibilidad de la máquina que recibe la información y la particularidad del individuo (ser humano), lo cual hace de este proceso un constructo que sólo puede integrar otro ser humano, y que escapa actualmente a nuestra imaginación del futuro próximo, en el cual, posiblemente se pueda incluso con un computador y una resonancia funcional lograr una aproximación a un diagnóstico diferencial incluyendo en el análisis emocional del paciente. Esto no está lejos de la realidad así como no lo es para los estudiantes el tener la facilidad de viajar y compartir experiencias con todo el mundo en un solo instante sin tener que esperar al paso de los años para conocerlo... es decir, tienen el acceso al conocimiento del mundo de una manera diferente y mucho más fácil que el que teníamos hace un tiempo.

Todo esto es lo que ha influido de manera significativa en las nuevas generaciones quienes tienen claridad en cuanto a que "no es necesario saberlo todo", sino encontrar la forma de hallar el conocimiento de manera efectiva. Este es un paradigma que rompieron las nuevas generaciones y nos cuesta mucho trabajo entender. Lo importante para nosotros como docentes consiste en encontrar el punto en el cual se entienda que no todo es tan simple como saber acceder a la información. Entonces ¿qué se debe aprender significativamente? Es allí donde debemos comenzar el trabajo como educadores, la idea es pensar no solamente en qué debe saber sino cómo le ayudo a asociar este conocimiento con otros de tal manera que lo logre integrar a sus conceptos y sea importante o relevante en el momento de razonar médicamente.

Este proceso requiere motivación, pero no es una motivación como se daba anteriormente, con elogio en público o dando buenas notas o exaltando un trabajo, no, la motivación debe ser personal, entonces, esto solamente lo logramos con un ejemplo personal, mostrando el gusto por el estudio y la práctica profesional, generando espacios de contacto interactivo con el estudiante ¿Qué estrategias didácticas debo emplear para que el estudiante se sienta participativo de su pro- 
ceso de aprendizaje y prefiera esta dinámica a tener que simplemente leer un libro sin ningún tipo de motivación personal diferente al ser evaluado con un cuestionario de respuesta múltiple? ¿Qué hago para que el estudiante se mantenga interactivo con el conocimiento?

La memoria repetitiva pasa a un segundo plano si no existe una asociación conceptual. El saber porqué y para qué debo aprender algo, es fundamental para el estudiante actual debido a la excesiva información con la que cuenta y por lo tanto piensa que, leer más de aquello que no tenga utilidad práctica, es perder el tiempo. Es curioso que el querer saber por qué y para qué, sea importante para los alumnos a quienes consideramos, no tienen la madurez necesaria para este cuestionamiento, pero esto es también cambiante en esta generación puesto que este es un concepto de aplicación de la andragogía (educación de los adultos). Volviendo al punto anterior, lo que debemos pensar es cómo evitar dar grandes contenidos de lecturas, sin priorizar los conceptos dentro de las mismas (lo cual es un error frecuente) y cómo no pretender dar todo el conocimiento de la asignatura a pesar de no ser pertinente para el nivel que se espera. Este pensamiento reflexivo acerca de cuándo, cuánto, con qué frecuencia debe enfrentar al estudiante el conocimiento es lo que pretende la secuenciación curricular. No todo puede ser en un solo momento, sin práctica, sin aplicación, de memoria.

Anteriormente se consideraba un criterio de calidad de enseñanza el dar grandes contenidos de gran profundidad de las diversas asignaturas olvidando incluso el objeto del conocimiento, en nuestro caso, el paciente o la salud; por esto muchas veces la experiencia era considerada en un segundo plano y mucho menos el integrar el concepto a otras disciplinas. Todo esto debe cambiar, el objeto es el paciente y su salud por lo tanto no debemos olvidar el facilitar el aprendizaje del estudiante alrededor del mismo ojalá con un contacto temprano con el paciente y sus problemáticas con una visión integral y no "por partes", como se hacía en la enseñanza tradicional. El cambio actual consiste en tener una visión sistémica de la enseñanza en medicina, inter y transdisciplinar, tratando de integrar las disciplinas, teniendo muy claro el objeto final de formación (característico de un currículo integrado), sin considerar que sea el maestro en quien se deba centrar la educación; la educación se centra en el estudiante y por lo tanto la prioridad no la tienen los procesos de enseñanza sino, el aprendizaje. Esto hay que entenderlo bien, no significa que el profesor no sea importante, todo lo contrario, su valor radica en que, de él depende el "cuánto aprendieron", y por lo tanto, la educación no se centra como antes se hacía, en la certeza acerca de "cuánto sabe el docente".

Cada vez toma mayor importancia en la educación médica el tener en cuenta la formación integral del estudiante entendido como individuo, social, personal, profesional, humano, espiritual y es por ello que dista de la generación de un profesional con énfasis solamente en sus habilidades cognitivas y técnicas. Si bien esto es parte fundamental del proceso, la educación médica tiende a incluir en sus propósitos, una formación en actitudes, en comunicación, en conocimiento de la bioética y la aplicación de sus principios en su comportamiento e inves- 
tigación, en la toma de decisiones, el pensamiento crítico y analítico, en el trabajo en equipo, el liderazgo y el manejo de comunidades, entre otros.

Todo esto es complejo de hacer evidente pero es allí donde debemos trabajar curricularmente, para describir lo que esperamos desde estos puntos de vista y el camino que debemos seguir para llegar a la formación de dichas competencias (competencia entendida como una acción, un sujeto u objeto sobre el que recae la acción y un nivel de calidad de la acción esperada). Además el ver la necesidad de formación en campos como la prevención de la enfermedad, la promoción de la salud, la administración y gestión nos cambian las perspectivas estratégicas de formación para estos campos. Lo anteriormente expuesto es lo que ha generado una gran revolución académica en el mundo, haciendo reformas curriculares acordes con el contexto actual del ejercicio profesional.

La medicina ha sido tradicionalmente enseñada utilizando al paciente como instrumento de aprendizaje, si bien esto es inmodificable porque es el objeto de estudio y es solamente en él en quien se adquiere la experiencia y el conocimiento profesional, existe una gran dificultad para abordarlo en iguales condiciones que hace unos años para la educación y actualmente el paciente no permite la práctica de los estudiantes de igual manera que antes, se cuenta con menor tiempo de contacto con el paciente, los pacientes son tratados cada vez más de manera ambulatoria, la continuidad del cuidado del paciente es muy difícil de llevar a cabo en nuestro sistema de atención en salud, el seguir la evolución del caso con límites de tiempo en el trabajo con el paciente y en fin, la enseñanza con el paciente ha cambiado por todo lo descrito pero ha generado errores que trascienden en la salud del paciente; es por ello que otro punto central en la educación médica actual es: la "seguridad del paciente".

Es allí donde se genera la necesidad de cambio en las estrategias necesarias para el aprendizaje del estudiante, es allí precisamente donde la simulación clínica ha tomado un gran valor en la enseñanza de la medicina, porque da seguridad en el ambiente de aprendizaje, facilita el hecho de poder mostrar múltiples problemas de los pacientes ( $\sin$ tener que esperarlos al azar o con el paso del tiempo); favorece el desarrollo de habilidades facilitando el aprendizaje con ensayo y error aprendiendo de estos últimos de una manera positiva y dando una mayor confianza al estudiante cuando enfrenta al paciente de manera real (teoría denominada: seguridad psicológica); puede además aprender viendo sus errores en escenarios simulados a través de análisis reflexivo posterior (Debriefing, término anglosajón aplicado a la reconstrucción de los hechos con aprendizajes de los mismos) y un valor de fondo que se aplica a través de la simulación es, la expresión del ciclo de Kolb del aprendizaje, en el cual vemos que es necesaria la experimentación, luego la reflexión, la conceptualización y de nuevo la experiencia para lograr un aprendizaje perdurable. De igual manera desarrolla el aprendizaje emocional (emotional learning), cuyo estudio es amplio en la actualidad y tiene gran importancia dadas las necesidades descritas de contexto.

Todo lo descrito nos orienta a la necesidad de hacer transformaciones curriculares, implementando sistemas educativos en los cuales predomine la par- 
ticipación interactiva del estudiante, el uso de nuevas tecnologías educativas, de la informática, el chat, el video, audio, y otros más; nos lleva entonces a pensar en la necesidad de implementar nuevos sistemas evaluativos por competencias, dando relevancia al uso del portafolio como instrumento importante en la evaluación por procesos, evaluando por medio de OSCE (evaluación objetiva clínica estructurada), utilizando técnicas de triple salto, o específicas de observación clínica, listas de chequeo y tantas otras técnicas de evaluación con las cuales contamos en la actualidad; es por ello que debemos generar espacios curriculares para la evaluación formativa, evaluación como proceso (que es muy diferente a lo que se aplica como momentos de evaluación), y considerar el hecho de permitir espacios para la auto, hetero y co-evaluación.

En fin, son muchas las ideas que nos pueden venir a la mente cuando pensamos en reforma curricular. Son múltiples las limitaciones que podemos tener para realizar nuestros propósitos de formación y mucho más dependiendo de los sistemas de atención en salud, pero no podemos permitir que el tiempo pase y cambie nuestro entorno sin dar un aporte positivo a la transformación de los procesos educativos que deben contribuir a formar profesionales con nuevas herramientas para los nuevos entornos. 